\title{
The Effect of Patient Observation on Cranial Computed Tomography Rates in Children with Minor Head Trauma
}

Sonia Singh, MD; ${ }^{1}$ Stephen J.C. Hearps, MBiostat; ${ }^{2}$ Meredith L. Borland, MBBS; ${ }^{3}$ Stuart R. Dalziel, PhD; ${ }^{4}$ Jocelyn Neutze, MBChB; ${ }^{5}$ Susan Donath, MA; ${ }^{6}$ John A. Cheek, MBBS $;{ }^{7}$ Amit Kochar, MD $;{ }^{8}$ Yuri Gilhotra, MBBS $;{ }^{9}$ Natalie Phillips, MBBS; ${ }^{10}$ Amanda Williams, BN; ${ }^{11}$ Mark D. Lyttle, MBChB; ${ }^{12}$ Silvia Bressan, PhD; ${ }^{13}$ Jeffrey S. Hoch, $\mathrm{PhD} ;{ }^{14}$ Ed Oakley, MBBS; ${ }^{15}$ James F. Holmes, MD; ${ }^{16}$ Nathan Kuppermann, MD; ${ }^{17}$ and

Franz E Babl, MD; ${ }^{18}$ on behalf of the Paediatric Research in Emergency Departments International Collaborative (PREDICT)

\section{Authors}

1. Sonia Singh, MD

singhsd@student.unimelb.edu.au

Murdoch Children's Research Institute, Melbourne, VIC, Australia

Address: 50 Flemington Rd, Parkville, VIC, 3052, Australia.

Department of Paediatrics, Faculty of Medicine, Dentistry and Health Sciences,

University of Melbourne, Melbourne, VIC, Australia

Address: Grattan St, Parkville, VIC, 3010, Australia.

University of California Davis Medical Center, Sacramento, CA, USA

Address: Department of Emergency Medicine, UCDMC, 2315 Stockton Blvd. PSSB

2100, Sacramento, CA 95817, USA

2. Stephen J.C. Hearps, MBiostat

stephen.hearps@mcri.edu.au

Murdoch Children's Research Institute, Melbourne, VIC, Australia

Address: 50 Flemington Rd, Parkville, VIC, 3052, Australia.

3. Meredith L. Borland, MBBS

This is the author manuscript accepted for publication and has undergone full peer review but has not been through the copyediting, typesetting, pagination and proofreading process, which may lead to differences between this version and the Version of Record. Please cite this article as doi: $10.1111 /$ ACEM.13942

This article is protected by copyright. All rights reserved 
meredith.borland@,health.wa.gov.au

Perth Children's Hospital, Perth, WA, Australia

Address: Emergency Department, Perth Children's Hospital, 15 Hospital Avenue,

Nedlands, Perth, WA, 6009, Australia

Divisions of Paediatrics and Emergency Medicine, School of Medicine University of Western Australia.

Address: 35 Stirling Hwy, Crawley, WA, 6009, Australia.

4. Stuart R. Dalziel, PhD

sdalziel@adhb.govt.nz

Starship Children's Health, Auckland, New Zealand

Address: Emergency Department, Starship Children's Health, 2 Park Rd, Grafton, Auckland, 1023, New Zealand.

Departments of Surgery and Paediatrics: Child and Youth Health, University of

Auckland, Auckland, New Zealand

Address: 2 Park Rd, Grafton, Auckland, 1023, New Zealand.

5. Jocelyn Neutze, MBChB

jocelyn.neutze@middlemore.co.nz

Kidzfirst Middlemore Hospital, Auckland, New Zealand

Address: Emergency Department, Kidzfirst Middlemore Hospital, 100 Hospital Rd, Auckland, 2025, New Zealand.

6. Susan Donath, MA

susan.donath@mcri.edu.au

Murdoch Children's Research Institute, Melbourne, VIC, Australia

Address: 50 Flemington Rd, Parkville, VIC, 3052, Australia.

Department of Paediatrics, Faculty of Medicine, Dentistry and Health Sciences, University of Melbourne, Melbourne.

Address: Grattan St, Parkville, VIC, 3010, Australia.

7. John A. Cheek, MBBS

This article is protected by copyright. All rights reserved 
john.cheek@rch.org.au

Murdoch Children's Research Institute, Melbourne, VIC, Australia

Address: 50 Flemington Rd, Parkville, VIC, 3052, Australia.

Royal Children's Hospital, Melbourne, VIC, Australia

Address: Emergency Department, Royal Children's Hospital, 50 Flemington Rd,

Parkville, VIC, 3052, Australia.

Department of Paediatrics, Faculty of Medicine, Dentistry and Health Sciences,

University of Melbourne, Melbourne, VIC, Australia

Address: Grattan St, Parkville, VIC, 3010, Australia.

8. Amit Kochar, MD

amit.kochar@sa.gov.au

Women's \& Children's Hospital, Adelaide.

Address: Emergency Department, Women's \& Children's Hospital, 72 King William

Road, North Adelaide, SA, 5006, Australia

9. Yuri Gilhotra, MBBS

yurigilhotra@me.com

Queensland Children's Hospital, Brisbane, QLD, Australia

Address: Emergency Department, Queensland Children's Hospital, 501 Stanley St, South Brisbane, QLD, 4101, Australia.

10. Natalie Phillips MBBS

natalie.phillips@merton-oxford.com

Queensland Children's Hospital, Brisbane and Child Health Research Centre, School of Medicine, The University of Queensland, QLD Australia

Address: Emergency Department, Queensland Children's Hospital, 501 Stanley St, South Brisbane, QLD, 4101, Australia.

11. Amanda Williams, BN

amanda.williams@rch.org.au 
Murdoch Children's Research Institute, Melbourne, VIC, Australia

Address: 50 Flemington Rd, Parkville, VIC, 3052, Australia.

12. Mark D Lyttle, MBChB

mdlyttle77@gmail.com

Murdoch Children's Research Institute, Melbourne, VIC, Australia

Address: 50 Flemington Rd, Parkville, VIC, 3052, Australia.

Bristol Royal Hospital for Children, Bristol, UK

Address: Emergency Department, Bristol Children's Hospital, Paul O'Gorman Building, Upper Maudlin St, Bristol, BS2 8BJ, United Kingdom.

Academic Department of Emergency Care, University of the West of England, Bristol.

Address: Blackberry Hill, Bristol, BS16 1XS, United Kingdom.

\section{Silvia Bressan, $\mathrm{PhD}$}

silviabress@gmail.com

Murdoch Children's Research Institute, Melbourne, VIC, Australia

Address: 50 Flemington Rd, Parkville, VIC, 3052, Australia.

Department of Women's and Children's Health, University of Padova, Padova, Italy

Address: Via Giustiniani3, 2 35128, Padova, PD, Italy.

14. Jeffrey S. Hoch, PhD

jshoch@ucdavis.edu

Center for Healthcare Policy and Research, University of California at Davis, Sacramento, California, USA

Address: 2103 Stockton Blvd., Sacramento, CA 95817, USA

Division of Health Policy and Management, Department of Public Health Sciences, University of California at Davis, Davis, California, USA

Address: One Shields Ave., Medical Sciences 1-C, University of California at Davis, Davis, CA 95616, USA

\section{Ed Oakley, MBBS}

This article is protected by copyright. All rights reserved 
ed.oakley@rch.org.au

Murdoch Children's Research Institute, Melbourne, VIC, Australia

Address: 50 Flemington Rd, Parkville, VIC, 3052, Australia.

Department of Paediatrics, Faculty of Medicine, Dentistry and Health Sciences, University of Melbourne, Melbourne, VIC, Australia

Address: Grattan St, Parkville, VIC, 3010, Australia.

Royal Children's Hospital, Melbourne, VIC, Australia

Address: Critical Care Medicine, Royal Children's Hospital, 50 Flemington Rd,

Parkville, VIC, 3052, Australia.

16. James F. Holmes, MD

jfholmes@ucdavis.edu

University of California, Davis School of Medicine, Sacramento, VA, USA

Address: Department of Emergency Medicine, 2315 Stockton Blvd. PSSB 2100

Sacramento, CA 95817, USA

Center for Healthcare Policy and Research, University of California at Davis, Sacramento, California, USA

Address: 2103 Stockton Blvd., Sacramento, CA 95817, USA

17. Nathan Kuppermann, MD

nkuppermann@ucdavis.edu

University of California, Davis School of Medicine, Sacramento, CA, USA

Address: Department of Emergency Medicine, 2315 Stockton Blvd. PSSB 2100

Sacramento, CA 95817, USA

Department of Pediatrics, University of California Davis School of Medicine,

Sacramento, CA, USA

Address: 2516 Stockton Blvd, Sacramento, CA 95817, USA

18. Franz E. Babl, MD

franz.babl@rch.org.au

Murdoch Children's Research Institute, Melbourne, VIC, Australia 
Address: 50 Flemington Rd, Parkville, VIC, 3052, Australia.

Department of Paediatrics, Faculty of Medicine, Dentistry and Health Sciences,

University of Melbourne, Melbourne, VIC, Australia

Address: Grattan St, Parkville, VIC, 3010, Australia.

Royal Children's Hospital, Melbourne, VIC, Australia

Address: Emergency Department, Royal Children's Hospital, 50 Flemington Rd, Parkville, VIC, 3052, Australia.

Affiliations: From the Murdoch Children's Research Institute, Melbourne, Australia (Singh, Hearps, Donath, Cheek, Williams, Lyttle, Bressan, Oakley, Babl); Department of Paediatrics, University of Melbourne, Australia (Singh, Donath, Cheek, Oakley, Babl); University of California Davis Medical Center, Sacramento, CA, USA (Singh); Perth Children's Hospital, Perth, Australia and Divisions of Paediatrics and Emergency Medicine, School of Medicine, University of Western Australia, Australia (Borland); Starship Children's Health, Auckland, New Zealand and Departments of Surgery and Paediatrics: Child and Youth Health, University of Auckland, Auckland, New Zealand (Dalziel); Kidzfirst Middlemore Hospital, Auckland (Neutze); Royal Children's Hospital, Melbourne, Australia (Cheek, Oakley, Babl); Women's \& Children's Hospital, Adelaide, Australia (Kochar); Queensland Children's Hospital, Brisbane, Australia (Gilhotra, Phillips); Child Health Research Centre, University of Queensland, Brisbane, Australia (Phillips); Bristol Royal Hospital for Children, Bristol, UK and Academic Department of Emergency Care, University of the West of England, Bristol, UK (Lyttle); Department of Women's and Children's Health, University of Padova, Padova, Italy (Bressan); Division of Health Policy and Management, Department of Public Health Sciences, University of California at Davis, Davis, California, USA (Hoch); Center for Healthcare Policy and Research, University of California at Davis, Sacramento, California, USA (Hoch, Holmes); Department of Emergency Medicine, University of California, Davis School of Medicine, Sacramento, CA, USA (Kuppermann, Holmes); Department of Pediatrics, University of California Davis School of Medicine, Sacramento, CA, USA (Kuppermann). 
Author contributions: SS conceived the study, interpreted the data and wrote the initial draft of the article. All authors designed the study, approved publication, and agreed to be accountable for all aspects of the work. MLB, SRD, JN, JAC, AK, YG, NP, AW, MDL, $\mathrm{SB}, \mathrm{EO}$, and FEB obtained the data. SJCH and FEB had full access to all the data in the study and take responsibility for the integrity of the data. SJCH takes responsibility for the accuracy of the data analysis. SS, SJCH, JCH, SD, JFH, NK, and FEB supervised the analysis of the data, contributed to the interpretation of the data, and revised the article critically. MLB, SRD, JN, JAC, AK, YG, NP, AW, MDL, SB, EO, JFH, NK, and FEB interpreted the data, provided supervision, and drafted or revised the paper critically. SS takes responsibility for the paper as a whole.

All authors attest to meeting the four ICMJE.org authorship criteria: (1) Substantial contributions to the conception or design of the work; or the acquisition, analysis, or interpretation of data for the work; AND (2) Drafting the work or revising it critically for important intellectual content; AND (3) Final approval of the version to be published; AND (4) Agreement to be accountable for all aspects of the work in ensuring that questions related to the accuracy or integrity of any part of the work are appropriately investigated and resolved.

\section{Corresponding Author: Sonia Singh}

Email singhsd@student.unimelb.edu.au

Running Title: Patient Observation and Cranial Computed Tomography Use

Keywords: planned observation, cranial CT, PECARN TBI risk group, clinically important traumatic brain injury

\section{Word Count: 4385}

Prior Presentations: Presented in part at the Pediatric Academic Societies meeting, April 2019, Baltimore, MD; Society for Academic Emergency Medicine, May 2019, Las 
Vegas, NV; 18th International Conference on Emergency Medicine, June 2019, Seoul, Korea

\section{Funding Sources/Disclosures:}

Funding and support: The study was funded by grants from the National Health and Medical Research Council (project grant GNT1046727, Centre of Research Excellence for Pediatric Emergency Medicine GNT1058560), Canberra, Australia; the Murdoch Children's Research Institute, Melbourne, Australia; the Emergency Medicine Foundation (EMPJ-11162), Brisbane, Australia; Perpetual Philanthropic Services (2012/1140), Australia; Auckland Medical Research Foundation (No. 3112011) and the A + Trust (Auckland District Health Board), Auckland, New Zealand; WA Health Targeted Research Funds 2013, Perth, Australia; the Townsville Hospital and Health Service Private Practice Research and Education Trust Fund, Townsville, Australia; and supported by the Victorian Government's Infrastructure Support Program, Melbourne, Australia. SS is supported by an Australian Government Research Training Program scholarship, and a PREDICT CRE Research Higher Degree scholarship. FEB's time was partly funded by a grant from the Royal Children's Hospital Foundation and the Melbourne Campus Clinician Scientist Fellowship, Melbourne, Australia, and an NHMRC Practitioner Fellowship, Canberra, Australia. SRD's time was partly funded by the Health Research Council of New Zealand (HRC13/556).

Clinical Trial Registration: Australian New Zealand Clinical Trials Registry (ANZCTR) ACTRN12614000463673

Declaration of conflicts of interest: None of the authors have conflicts of interest.

Financial Disclosure: The authors have no financial relationships relevant to this article to disclose.

\section{Acknowledgments:}

We thank the participating families and emergency department staff at participating sites. We thank Sarah Dalton and Mary McCaskill (The Children's Hospital at Westmead, Sydney); Jeremy Furyk (The Townsville Hospital, Townsville); and Louise Crowe (Murdoch Children's Research Institute, Melbourne) for their involvement with obtaining 
the data and prior data analysis. We thank Daniel Tancredi, Ph.D. (Departments of Pediatrics and Emergency Medicine at the University of California Davis School of Medicine, Sacramento, CA, USA) for his review of the statistical analysis; and Amrita Singh (Brown University, Providence, RI, USA) for critical review of the manuscript.

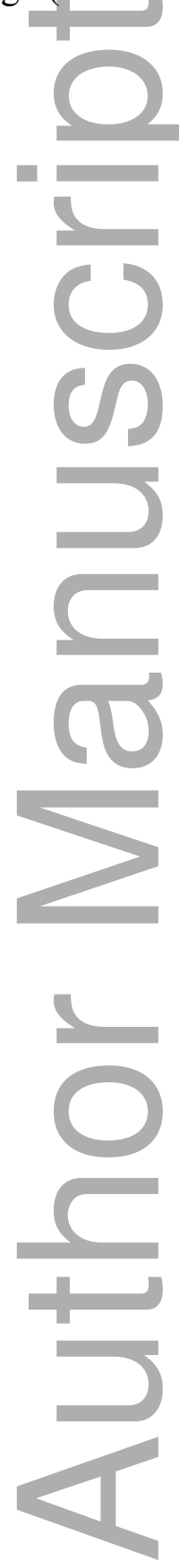


Patient Observation and Cranial Computed Tomography Use

2 DR. SONIA SINGH (Orcid ID : 0000-0003-2430-0262)

ABSTRACT

9 Background: Management of children with minor blunt head trauma often includes a period of 10 observation to determine the need for cranial computed tomography (CT). Our objective was to 11 estimate the effect of planned observation on CT use for each Pediatric Emergency Care Applied

12 Research Network (PECARN) traumatic brain injury (TBI) risk group among children with 13 minor head trauma.

14 Methods: This was a secondary analysis of a prospective observational study at 10 emergency 15 departments (EDs) in Australia and New Zealand, including 18,471 children <18 years-old, 16 presenting within 24 hours of blunt head trauma, with GCS scores of 14-15. The planned 17 observation cohort was defined by those with planned observation and no immediate plan for 18 cranial CT. The comparison cohort included the rest of the patients, who were either not 19 observed or for whom a decision to obtain a cranial CT was made immediately after ED 20 assessment. The outcome clinically important TBI (ciTBI) was defined as death due to head 21 trauma, neurosurgery, intubation for $>24$ hours for head trauma, or hospitalization for $\geq 2$ nights 22 in association with a positive cranial CT scan. We estimated the odds of cranial CT use with 23 planned observation, adjusting for patient characteristics, PECARN TBI risk group, history of 24 seizure, time from injury, and hospital clustering, using a generalized linear model with mixed 25 effects.

26 Results: The cranial CT rate in the total cohort was $8.6 \%$, and $0.8 \%$ had ciTBI. The planned 27 observation group had 4,945 (27\%) children compared to $13,526(73 \%)$ in the no planned 28 observation group. Cranial CT use was significantly lower with planned observation (adjusted 29 odds ratio [OR] 0.2, [95\% CI: 0.1-0.1]), with no difference in missed ciTBI rates. There was no 30 difference in the odds of cranial CT use with planned observation for the group at very low risk 31 for ciTBI (adjusted OR 0.9, [95\% CI: 0.5-1.4]). Planned observation was associated with 
32 significantly lower cranial CT use in patients at intermediate-risk (adjusted OR 0.2, [95\%CI: 0.2-

33 0.3]) and high-risk (adjusted OR 0.1, [95\% CI: 0.0-0.1]) for ciTBI.

34 Conclusion: Even in a setting with low overall cranial CT rates in children with minor head 35 trauma, planned observation was associated with decreased cranial CT use. This strategy can be 36 safely implemented on selected patients in the PECARN intermediate and higher-risk groups for 37 ciTBI.

\section{INTRODUCTION}

56 Minor head trauma in children is a common emergency department (ED) presentation and is

57 frequently defined by a Glasgow Coma Scale (GCS) score of 14-15 following head trauma. ${ }^{1,2}$

58 Cranial computed tomography (CT) to diagnose traumatic brain injury (TBI) after trauma is the 59 current gold standard investigation, however, the ionizing radiation exposure is associated with 60 an increased risk of lethal malignancies. ${ }^{3-6}$ There has been concern about increasing CT rates in 61 children presenting to EDs in the United States from 1998 to 2008 . $^{7,8}$ Retrospective studies on 62 cranial CT trends in pediatric EDs have noted considerably higher rates in the U.S. (27.6\% in 
632007 to $30.2 \%$ in 2015$)^{9}$ compared with Australia (9.5\% in 2001 to $9.6 \%$ in 2010). ${ }^{10}$ This

64 difference has been confirmed with prospective studies in children reporting lower non-risk65 adjusted cranial CT rates in Australia (8.3\%) compared to the U.S. (35.3\%), with similar rates of 66 clinically important traumatic brain injuries (ciTBIs). ${ }^{111}$ Clinical decision rules (CDRs) have 67 been developed and validated, to aid clinical decision-making, improve the sensitivity of 68 identifying patients with TBI and optimize cranial CT rates by decreasing unnecessary CT

71 With the Choosing Wisely campaign, the American Academy of Pediatrics and the American 72 College of Emergency Physicians have endorsed the benefit of an observation period for the 73 management of pediatric head injury. ${ }^{15,16}$ Observation reduces cranial CT scan rates for children 74 with minor blunt head trauma, with no significant impact on the delayed diagnosis of ciTBI. ${ }^{17,18}$ 75 One investigation of 40,113 children reported that observation was associated with lower cranial 76 CT use when compared with those not observed (adjusted odds ratio [OR] 0.53). ${ }^{17}$ In a 77 subsequent study of 1,605 patients, every hour increase in ED observation was associated with 78 decreased cranial CT rates across all 3 risk groups of children with minor head trauma at risk for 79 ciTBI in the clinical decision rule derived by the Pediatric Emergency Care Applied Research 80 Network (PECARN): very low-risk (adjusted OR 0.47), intermediate-risk (adjusted OR 0.28), 81 and high-risk (adjusted OR 0.11). ${ }^{18}$

83 We explored the relationship between planned ED observation and cranial CT use in children 84 with minor blunt head trauma in a clinical setting with low baseline cranial CT rates. 85 Additionally, we stratified patients according to their risk for ciTBI and estimated the effect of 86 ED observation on CT rates for children in the different PECARN-defined TBI risk groups

87 (Table 1) and the impact of observation on missed ciTBI rates. ${ }^{1}$

88

\section{METHODS}

\section{Study Design}

91 This was a planned secondary analysis of a prospective cohort study of children younger than 18 92 years of age with minor blunt head trauma, enrolled between April 2011 and November 2014 in 9310 pediatric EDs in Australia and New Zealand which are members of the Pediatric Research in 
94 Emergency Departments International Collaborative (PREDICT) network. ${ }^{19}$ The primary study 95 was designed to validate 3 clinical head injury decision rules: PECARN (USA), CATCH 96 (Canada), and CHALICE (UK). ${ }^{1,12,13,20}$ The individual hospital ethics committee for each 97 participating site approved the study. The details of the study protocol have been previously 98 published. ${ }^{20}$

\section{Study Setting and Population}

100 The participating EDs were a combination of 2 mixed and 8 free-standing children's hospital 101 EDs across Australia and New Zealand. The treating clinicians enrolled patients presenting to the 102 ED with blunt head trauma. A standardized case report form was completed recording 103 demographic, epidemiologic, and clinical information. After the initial ED assessment, clinicians 104 documented responses to two questions that were not mutually exclusive: if they planned to 105 observe the patient ("Do you intend to observe the patient in ED for head injury? yes/no"), and if 106 they planned to obtain immediate neuroimaging ("Do you intend to perform neuroimaging for 107 this patient? yes/no") -- if yes, ("What neuroimaging do you intend to perform? CT, MRI, US, 108 Skull X-ray"). Based on the responses, the planned observation cohort included those with 109 planned observation and no immediate plan for cranial CT. The comparison cohort included the 110 rest of the patients, who were either not observed or for whom a decision to obtain a cranial CT 111 was made immediately after ED assessment. Planned ED observation was defined as "yes" to the 112 question, "Do you intend to observe the patient in ED for head injury?"

\section{Study Protocol}

114 Inclusion criteria: All children younger than 18 years of age presenting to the ED after sustaining 115 blunt head trauma of any severity were enrolled in the primary study. ${ }^{11}$ For this sub-analysis, we 116 selected the cohort of children with GCS scores of 14-15 presenting within 24 hours of head 117 trauma, to enable direct comparison with prior published results. Children with multiple ED 118 visits, with different episodes of head trauma, were eligible for enrollment at each visit.

119 Exclusion criteria: Published in the primary study, ${ }^{11}$ which included patients with trivial facial 120 trauma, if they presented to the ED with neuroimaging for head trauma before study enrollment, 121 and those with history of blunt head trauma who left the ED without being seen by a physician.

\section{Study Measures}

123 Physicians recorded ED data, and research assistants recorded the data regarding hospital 124 management and conducted structured telephone follow-up on all patients who did not get 
125 neuroimaging in the ED 14-90 days after the initial ED visit. Results of cranial CTs performed 126 on patients who re-presented to the ED were reviewed by the site investigators. Neuroimaging 127 and neurosurgery reports were reviewed for any patients identified on the follow-up telephone 128 call to have had medical visits outside the original study hospital. Patients who were admitted to 129 the hospital or discharged from ED after a cranial CT was obtained, did not receive a follow-up 130 phone call.

132 All site investigators and research coordinators were trained at the central site prior to 133 commencing the study. Site clinicians and research assistants were trained by the site 134 investigators and research coordinators using standardized teaching materials in 1-hour sessions. 135 This was repeated as needed for new staff throughout the study period. Site investigators and 136 research coordinators participated in annual updates. The central study coordinator conducted 137 annual site visits for oversight, compliance, and ongoing updates of site personnel. ${ }^{20}$

139 Timing of events was calculated in hours using information collected on case report forms, 140 including time from injury to ED arrival, time from ED arrival to physician evaluation, ED 141 length of stay (LOS), and total LOS. We defined ED LOS as the interval of time from ED arrival 142 until ED discharge. Data on admission rates included those admitted to short-stay units for 143 observation, hospital wards, or pediatric intensive care units (PICU). The short-stay unit 144 admitted patients with anticipated LOS <24 hours. The location of the short-stay units varied 145 across the participating hospitals (part of ED, separate unit in ED, or a hospital ward). As a 146 result, for those patients admitted to observation units, but not the hospital wards or PICU, the 147 ED LOS was the interval of time from ED arrival until hospital discharge. Total LOS was 148 defined as the time from ED arrival to hospital discharge.

150 To minimize any potential selection bias between the two study cohorts, we stratified the 151 population by their risk for ciTBI using the age-based PECARN TBI risk groups: very low, 152 intermediate, and high (Table 1). ${ }^{1}$ Children with no predictors for ciTBI were classified as very 153 low risk. Children with 1 or more risk factors were classified as intermediate or high risk, 154 depending on the specific risk factor, according to the original PECARN paper. ${ }^{1}$ We analyzed 155 CT use for each of the PECARN TBI risk groups. 
Patient Observation and Cranial Computed Tomography Use

157 The primary outcome measures were CT and ciTBI rates. Secondary outcome measures were ED

158 and total LOS, rates of TBI on cranial CT, and hospital admission. The ED clinician's initial

159 assessment of the patient's GCS score, attending radiologist reports of the CT scans, and 160 operative reports of neurosurgical interventions were used for each site.

162 The outcome, cranial CT, was defined as present if the patient underwent the imaging study 163 during ED or short stay evaluation. The outcome, ciTBI, was defined as death as a result of 164 intracranial injury, neurosurgical intervention, intubation for longer than 24 hours for TBI or 165 hospital admission for 2 nights or longer in association with TBI on CT. ${ }^{1}$ Neurosurgical 166 intervention for TBI included intracranial pressure monitoring, elevation of depressed skull 167 fracture, ventriculostomy, hematoma evacuation, lobectomy, tissue debridement, dura repair, and 168 other procedures. ${ }^{1}$ Missed ciTBI was defined as the diagnosis of ciTBI on a follow-up ED visit 169 or identified on the follow-up phone call, with no cranial CT performed on the initial ED visit.

170 TBI on $\mathrm{CT}$ was defined as intracranial hemorrhage or contusion, cerebral edema, traumatic 171 infarction, diffuse axonal injury, shearing injury, sigmoid sinus thrombosis, midline shift of

172 intracranial contents or signs of brain herniation, diastasis of the skill, pneumocephalus, and skull 173 fracture depressed by at least the width of the table of the skull. ${ }^{1}$ The attending radiologists at 174 each hospital site were blinded to the study hypothesis and patient cohorts.

\section{Data Analysis}

176 The focus of this analysis was a comparison of the cohort with planned observation, and the 177 cohort who were either not observed or for whom a decision to obtain a CT was made 178 immediately after ED evaluation. We described these cohorts with population proportions and $17995 \%$ confidence intervals (CI). Additionally, we compared the distribution of patients within 180 each PECARN ciTBI risk group between the two cohorts. We performed a bivariate analysis of 181 rates of CT scans, ciTBI, missed ciTBI, TBI on CT, and hospital admission between the 2 182 cohorts, and for each PECARN TBI risk group. The proportion of patients with return ED visits 183 was calculated for those with follow-up phone calls.

185 We compared the time in hours as a categorical variable using median with interquartile range, 186 and rate difference using the Hodges-Lehmann method, which accounts for different population 
187 distributions in the two cohorts. ${ }^{21}$ The total LOS (hours) was compared, as well as ED LOS, LOS

188 for each PECARN TBI risk group, and LOS in patients who had cranial CT scans.

190 Using a generalized estimating equation model, we estimated cranial CT use with multivariable 191 logistic regression adjusting for patient demographics, PECARN TBI risk group, history of 192 seizure, and time from injury. History of seizure was not included as a risk factor for ciTBI in the 193 PECARN head injury rules. ${ }^{1}$ The investigators subsequently described the importance of 194 recurrent seizures as a risk factor associated with TBI on CT. ${ }^{22}$ In the current study, history of 195 seizure had a significant bivariate relationship with CT use. Therefore, it was included in the 196 multivariate analysis. Because planned observation and cranial CT rates could be correlated with 197 clinician practice within a hospital site, we controlled for hospital site as a random effect (with 198 random intercept). Furthermore, because clinician practice could vary across the study time199 period $(2011$ - 2014), we adjusted for the calendar year as a fixed effect, with 2011 as a

200 reference. We investigated variance inflation factor values to check for multicollinearity between 201 the variables, and model residuals and leverage to ensure the absence of outliers and influential 202 cases. Overall model performance was evaluated via assessment of classification and calibration 203 (prediction accuracy). Additionally, we performed a sensitivity analysis of the regression model 204 by adjusting for the individual signs and symptoms and mechanism of injury, instead of the 205 individual PECARN TBI risk groups. Finally, we estimated the adjusted odds (OR) of CT use 206 with planned observation for each PECARN TBI risk group.

208 Sample size calculations were performed and published in the primary study, ${ }^{11,20}$ and no 209 additional computations were performed for this planned sub-analysis. Additionally, there were 210 sufficient events in the outcome measure of cranial CT use (8.6\%, 1579 patients), such that 211 including the 7 covariates ensured a robust regression model. Data analysis was performed with 212 Stata (version 13; StataCorp, College Station, TX) for the analysis. This manuscript is reported 213 following the STROBE statement. ${ }^{23}$

216 Characteristics of study subjects 
217 Of the 20,137 enrolled patients in the primary study, ${ }^{24} 18,781(93.3 \%)$ presented within 24 hours 218 of sustaining head trauma, with GCS scores of 14-15 (Figure). Documented planned observation 219 and cranial CT status was available for 18,471 (91.7\%) of enrolled patients, and all subsequent 220 analyses were performed on this cohort. There were no differences in demographic 221 characteristics, cranial CT or ciTBI rates between the $310(1.5 \%)$ patients with no documented 222 observation plan and the analytic cohorts.

\section{Descriptive data}

225 The overall cranial CT rate was $8.6 \%$ (range 2-16\% across the different hospitals), with a ciTBI 226 rate of $0.8 \%$ and $0.1 \%$ of patients receiving a neurosurgical intervention. Cranial CT rates for 227 each of the PECARN TBI risk groups varied significantly as those at higher risk were more 228 likely to undergo CT: very low-risk group (1.8\% CT rate), intermediate-risk group (10.3\% CT 229 rate) and high-risk group (43.1\% CT rate). Similarly, the ciTBI rates varied significantly, with $2300.01 \%$ in the PECARN very low-risk group, $0.6 \%$ in the intermediate-risk and $6.3 \%$ in the high231 risk group.

233 The strategy of planned observation was made for 4,945 (26.8\%) patients (Table 2), with a range 234 of $21 \%$ to $49 \%$ across the participating hospitals. Those in the planned observation cohort were 235 slightly older and included $33.0 \%$ at very low risk, 54.9\% at intermediate risk, and $12.2 \%$ at 236 high-risk for ciTBI.

238 The median time from injury to ED arrival in the cohort with planned observation was not 239 statistically different from those with no observation (Table 3). The increase in ED LOS with 240 cranial CT use was significant with planned observation, and for each of the PECARN risk 241 groups. The total LOS was greater with planned observation for patients at very low-risk and 242 intermediate-risk, and lower for those at high-risk for ciTBI than those with no planned 243 observation.

\section{Main Results}

246 The unadjusted cranial CT rate was significantly lower in those with planned observation (4.4\%) 247 than those in the no planned observation cohort $(10.1 \%)$, as was the ciTBI rate $(0.4 \%$ vs. $0.9 \%$; 
Patient Observation and Cranial Computed Tomography Use

248

249

250

251

252

253

254

255

256

257

258

259

260

261

262

263

264

265

266

267

268

269

270

271

272

273

274

275

276

277

278

Table 2). There was a significant difference in the ward and PICU admission rates for the patients with planned observation (6.4\% and $0.3 \%)$ compared to those not observed (4.0\% and $0.1 \%$ ). There was no difference in the rates of missed ciTBI between the cohorts. Two patients in the intermediate PECARN risk group, both with planned observation, were admitted for ciTBI at the follow-up ED visit. Neither patient required neurosurgery. The details of these patients have been published. ${ }^{24}$ There were no deaths in either cohort.

\section{PECARN TBI Risk Groups}

There was no difference in the unadjusted cranial CT, ciTBI, and TBI on CT rates for the cohorts with and without planned observation, for the group at very low risk for ciTBI (Table 2). The planned observation group at intermediate risk for ciTBI had significantly lower CT use (4.4\%) than the group without planned observation $(13.3 \%)$, and there were no differences in rates of ciTBI $(0.5 \%$ vs. $0.6 \%)$ or TBI on CT $(0.7 \%$ vs. $1.1 \%)$. The planned observation group at high risk for ciTBI had significantly lower cranial CT use (11.7\%) than those without planned observation (62.5\%), with significantly lower rates of ciTBI (1.2\% vs. 9.4\%) and TBI on CT $(2.0 \%$ vs. $14.1 \%)$.

Multivariable Analysis

After adjusting for age, sex, PECARN TBI risk group, history of seizure, calendar year, and time from injury, the cohort with planned observation had significantly lower cranial CT use (adjusted OR 0.2, [95\% CI: 0.1-0.2]; Table 4). Additionally, there was no evidence of multicollinearity, with variance inflation factor of less than 1.5 for all variables in the model (except the nonindependent calendar years variable). A sensitivity analysis of the generalized linear model, adjusting for the mechanism of injury and individual signs and symptoms of TBI rather than PECARN risk group produced similar results (adjusted OR 0.2, [95\% CI: 0.1-0.2]; Supplement 1). Good model fit was found, and the classification rate was high (94\%; sensitivity $38 \%$, specificity 99\%). There was no difference in cranial CT use across calendar years for the total cohort and each individual PECARN TBI risk group.

Multivariate logistic regression for each of the PECARN TBI risk groups demonstrated that planned observation was significantly associated with lower CT use in patients at intermediaterisk (adjusted OR 0.2, [95\%CI: 0.2-0.3]) and high-risk (adjusted OR 0.1, [95\% CI: 0.0-0.1]) for ciTBI (Supplement 1). Good model fit was found, with high classification rates: high risk 83\% 
Patient Observation and Cranial Computed Tomography Use

279

280

281

282

283

284

285

286

287

288

289

290

291

292

293

294

295

296

297

298

299

300

301

302

303

304

305

306

307

308

309

(sensitivity $78 \%$, specificity $86 \%$ ); intermediate risk $90 \%$ (sensitivity $7.5 \%$, specificity $99 \%$ ).

The difference in cranial CT use between those with and without planned observation at very low risk for ciTBI was not significant (adjusted OR 0.9, [95\% CI: 0.5-1.4]), with a poor model fit.

\section{DISCUSSION}

This multicenter prospective study evaluated the strategy of planned ED observation and cranial CT use in children with minor head trauma. Despite the low baseline cranial CT rate, planned observation of patients was associated with $80 \%$ lower adjusted odds of CT use. Although the cohorts with and without planned observation were dissimilar in individual patient characteristics, after adjusting for patient characteristics, signs, symptoms, and risk factors for ciTBI, planned observation was independently associated with lower cranial CT use in all but those at very low risk of ciTBI.

The primary study protocol defined ED observation as an ongoing assessment for less than 6 hours after the initial clinical evaluation. Admission was defined as assessment requiring more than 6 hours to either an observation or short stay unit, inpatient or intensive care unit. ${ }^{20}$ Although there was no defined period of observation in the study protocol, in a subsequent analysis, a hospital length of stay greater than 4 hours was used as a proxy to evaluate the effect of observation. ${ }^{24}$ ED length of stay is affected by many factors that are often out of the clinician's control. Therefore, the impact of planned ED observation on CT use in patients for whom there was no plan for an immediate cranial $\mathrm{CT}$, provides the most accurate effect of time on clinical decision-making. The total LOS was greater for the cohort with no planned observation at high risk for ciTBI than those with planned observation. This was likely as a result of more children at high risk for ciTBI getting immediate cranial CTs $(62.5 \%)$, having TBI on CT $(14.1 \%)$, or ciTBI (9.4\%); either of which may have been associated with hospital admission.

Although the primary study of which this was a sub-analysis was designed to validate the existing head injury decision rules, clinicians did not report using any particular guideline for their decision-making. ${ }^{25}$ Additionally, with a stable cranial CT rate in the decade prior to the study, ${ }^{10}$ and no difference in cranial CT use across calendar years in the current study, suggests 
310 that the clinicians did not alter their practice during the study period. The comparison cohort was 311 comprised of patients with different risk profiles for ciTBI, consisting of those who were either

312 not observed (e.g., asymptomatic children with minor mechanisms of injury) or for whom a 313 decision to obtain a cranial CT was made right after ED evaluation (e.g., symptomatic children at 314 higher risk for TBI). This is similar to a prior study in which $72 \%$ of children at very high risk

315 for ciTBI were in the non-observed group. ${ }^{18}$ They reported a cranial CT rate of $5 \%$ in the patients 316 who were observed prior to the decision of imaging compared with $30 \%$ in the non-observed 317 cohort.

319 Stratifying the cohorts by the PECARN TBI risk groups allowed for a comparison of cranial CT 320 rates in patients at similar risk for ciTBI. There were no differences in the rates ciTBI and TBI 321 on CT for the very low and intermediate-risk patients between the two cohorts, indicating a 322 similar risk profile. After risk adjustment, however, planned ED observation was associated with 323 significantly lower cranial CT use in the groups at intermediate and high risk for ciTBI. The 324 group at very low risk for ciTBI who were observed did not have a statistically lower rate of 325 cranial CT use compared to those not observed. This is not surprising as cranial CT is not 326 recommended in this low-risk group, and therefore it would be unlikely that observation would 327 have much impact.

329 The results of this study validate the published literature from North America, where ED 330 observation is associated with lower cranial CT rates in children after minor blunt head 331 trauma. ${ }^{17,18}$ Prior research on the CT rates of each of the PECARN TBI risk predictors in the US 332 was reported as $13 \%$ of low risk, $54 \%$ of intermediate, and $82.5 \%$ of high-risk groups. $^{26}$ 333 Published evidence of the clinical practice in Australia and New Zealand for pediatric minor 334 head trauma termed as "usual care" report lower cranial CT rates than with the use of decision 335 rules. $^{10,24}$ Those publications report much lower CT rates for each of the PECARN TBI risk 336 groups (1.7\% of very low risk, $9.9 \%$ of intermediate, and $44 \%$ of high risk) with similar ciTBI 337 rates.

339 In addition to differences in clinical practice, this study highlights the use of short-stay units in 340 EDs in Australia and New Zealand. The Australian National Emergency Target (NEAT) or the 
341 4-hour rule was introduced in 2011, and a similar 6-hour target in New Zealand was

342 implemented in 2009, in response to ED overcrowding. ${ }^{27,28}$ These targets/rules require a certain

343 proportion of patients based on their triage acuity to be discharged, admitted or transferred

344 within 4 hours of ED presentation, with a potential loss of financial incentives when $90 \%$ of

345 targets are not met. Because admissions to short-stay units stop the NEAT target clock, they are

346 commonly used to decompress the ED. Perhaps developing similar short-stay units in pediatric

347 EDs in North America might aid with longer observation periods of children with minor head

348 trauma and result in fewer CT scans.

350 The increase in lifetime attributable risk of cancer associated with ionizing radiation of cranial

351 CT scans has been reported worldwide. ${ }^{4-6}$ The cumulative iatrogenic radiation in children might

352 triple the risk of leukemia and brain tumors, a large retrospective study reports. ${ }^{6}$ With increasing

353 awareness of these risks, it has become important to educate health care providers and parents

354 that CT decisions can often be undertaken as a shared decision-making process. ${ }^{29}$ Furthermore,

355 implementation of clinical decision tools has resulted in decreased CT rates for minor head

356 trauma at pediatric and general EDs. ${ }^{30-33}$ A recent study of 7 health systems in the US and

357 Ontario report a decreasing trend in cranial CT rates for children <18 years from 2006 to $2016 .^{8}$

358 However, this has not been the case at the national level in the US, with no apparent decrease in 359 cranial CT rates in children between 2007 and $2015 .{ }^{9}$

361 The PECARN age-based CDRs identify those patients at very low risk for ciTBI, and for whom

362 cranial CT is usually not recommended. ${ }^{1}$ Incorporating decision support in the electronic medical

363 record based on the PECARN rules reduces cranial CT rates in the ED for children at very low

364 risk for ciTBI. ${ }^{33}$ The average cranial CT rates across all the intervention EDs decreased from

$3655.3 \%$ to $4.2 \%$. Furthermore, it is possible to achieve overall cranial CT rates of $\sim 15 \%$ in all

366 children with minor blunt head trauma with GCS scores of $14-15 .^{33}$ We report no benefit from

367 planned observation in patients at very low risk for ciTBI, and cranial CT rate of $1.7 \%$, without

368 any missed ciTBI in this group. Following this analysis, we intend to evaluate the cost-

369 effectiveness of observation as a strategy to reduce CT use while minimizing missed ciTBI. 
371 To our knowledge, this is the first study to validate the impact of planned ED observation as a 372 strategy to lower cranial CT use for each PECARN TBI risk group, in a large cohort of children 373 with minor blunt head trauma. Despite the lower overall use of CT in this study, our findings are 374 in agreement with the previous study of planned observation conducted on a different

375 continent. ${ }^{17}$ Selective observation of patients with minor head trauma at intermediate risk of 376 ciTBI could provide time for resolution of symptoms and/or reassurance of the lack of ciTBI and 377 decrease the need for cranial CT. We found no increased risk of missed ciTBI in the observation 378 without a CT cohort, even among selected patients at higher risk of ciTBI. Therefore, this 379 strategy can be safely implemented on selected patients in the PECARN intermediate and higher380 risk groups for ciTBI.

\section{LIMITATIONS}

383 Our study has several limitations. First, the questions asked of the clinicians on the case report 384 forms regarding planned observation and planned immediate cranial CT were not mutually 385 exclusive. Additionally, the questions did not clarify the reasons for planned observation, and in 386 particular, the patients for whom the decision to obtain an immediate cranial CT was already 387 made. However, there was no difference in the cranial CT rates between these patients and those 388 who received an immediate cranial CT scan and were not observed. As a result, we believe the 389 effect of observation on the cranial CT rate is accurately reflected in the cohort with planned 390 observation and no plan for immediate cranial CT. Second, there were significant differences 391 between the patients with planned observation and those without planned observation. The 392 patients who received an immediate CT were more symptomatic, with frequent severe 393 mechanisms of injury, and at a higher risk for ciTBI than the cohort with planned observation.

394 Although we used multivariable logistic regression to adjust for risk factors of ciTBI to estimate 395 the independent effect of planned observation on cranial CT use, there could be some 396 unaccounted-for residual confounding or bias.

398 Third, we did not collect data on the time between physician evaluation to the time of the cranial 399 CT order. Therefore, we cannot measure the incremental effect of observation time on cranial CT 400 use. Further research in this area will guide the recommended length of ED observation. Finally, 401 because we did not have time stamps regarding the time of completion of case report forms, it is 
Patient Observation and Cranial Computed Tomography Use

402 not clear if the decision for neuroimaging was made because of the progression of symptoms or 403 other factors not recorded at the time the case report form was completed. As the data collected 404 were static, we do not know if the data accurately represent the dynamic nature of the evolution 405 of disease and clinicians' decision-making.

406

407 CONCLUSION

408 Planned observation, as a strategy for the management of minor blunt head trauma, is associated 409 with lower cranial CT use, even in a setting of low baseline CT rates. Children at very low risk 410 for ciTBI, do not benefit from observation as a strategy to lower cranial CT use. Our results 411 validate planned observation as an appropriate strategy for selected patients at intermediate and 412 higher risk for ciTBI, with lower adjusted odds of cranial CT use and no significant difference in 413 missed ciTBIs.

414

415

416

417

418

419

420

421

422

423

424

425

426

427

428

429

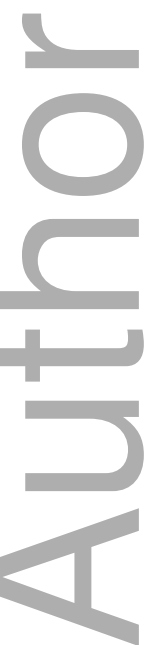

430 
Patient Observation and Cranial Computed Tomography Use

\section{REFERENCES}

434 1. Kuppermann N, Holmes JF, Dayan PS, et al. Identification of children at very low risk of

435 clinically-important brain injuries after head trauma: a prospective cohort study. Lancet

436 2009;374:1160-70.

437 2. Crowe LM, Hearps S, Anderson V, et al. Investigating the variability in mild traumatic brain 438 injury definitions: a prospective cohort study. Arch Phys Med Rehabil 2018;99:1360-9.

439 3. Brenner DJ, Hall EJ. Computed tomography - an increasing source of radiation exposure. $\mathrm{N}$ 440 Engl J Med 2007;357:2277-84.

441 4. Mathews JD, Forsythe AV, Brady Z, et al. Cancer risk in 680,000 people exposed to 442 computed tomography scans in childhood or adolescence: data linkage study of 11 million 443 Australians. BMJ 2013;346:f2360.

444 5. Miglioretti DL, Johnson E, Williams A, et al. The use of computed tomography in pediatrics 445 and the associated radiation exposure and estimated cancer risk. JAMA Pediatr 2013;167:700-7.

446 6. Pearce MS, Salotti JA, Little MP, et al. Radiation exposure from CT scans in childhood and

447 subsequent risk of leukaemia and brain tumours: a retrospective cohort study. Lancet 448 2012;380:499-505.

449 7. Larson DB, Johnson LW, Schell BM, Goske MJ, Salisbury SR, Forman HP. Rising use of CT 450 in child visits to the emergency department in the United States, 1995-2008. Radiology $4512011 ; 259: 793-801$.

452 8. Smith-Bindman R, Kwan ML, Marlow EC, et al. Trends in use of medical imaging in US 453 health care systems and in Ontario, Canada, 2000-2016. JAMA 2019;322:843-56.

454 9. Burstein B, Upton JEM, Terra HF, Neuman MI. Use of CT for head trauma: 2007-2015. 455 Pediatrics 2018;142.

456 10. Oakley E, May R, Hoeppner T, et al. Computed tomography for head injuries in children:

457 change in Australian usage rates over time. Emerg Med Australas 2017;29:192-7.

458 11. Babl FE, Borland M, Phillips N, et al. Accuracy of PECARN, CATCH, and CHALICE head 459 injury decision rules in children: a prospective cohort study. Lancet 2017;389:2393-402.

460 12. Dunning J, Daly JP, Lomas JP, et al. Derivation of the children's head injury algorithm for 461 the prediction of important clinical events decision rule for head injury in children. Arch Dis 462 Child 2006;91:885-91. 
Patient Observation and Cranial Computed Tomography Use

463 13. Osmond MH, Klassen TP, Wells GA, et al. CATCH: a clinical decision rule for the use of 464 computed tomography in children with minor head injury. Can Med Assoc J 2010;182:341-8.

465 14. Pickering A, Harnan S, Fitzgerald P, Pandor A, Goodacre S. Clinical decision rules for 466 children with minor head injury: a systematic review. Arch Dis Child 2011;96:414-21.

467 15. American Academy of Pediatrics | Choosing wisely: ten things physicians and patients 468 should question. 2013. at Choosingwisely.org.

469 http://www.choosingwisely.org/societies/american-academy-of-pediatrics/. Published 2013.

470 Accessed December 1, 2018.)

471 16. American College of Emergency Physicians | Choosing wisely: ten things physicians and

472 patients should question. 2013. at Choosingwisely.org.

473 http://www.choosingwisely.org/societies/american-college-of-emergency-physicians/. Published

474 2013. Accessed July 1, 2019.)

475 17. Nigrovic LE, Schunk JE, Foerster A, et al. The effect of observation on cranial computed 476 tomography utilization for children after blunt head trauma. Pediatrics 2011;127:1067-73.

477 18. Schonfeld D, Fitz BM, Nigrovic LE. Effect of the duration of emergency department 478 observation on computed tomography use in children with minor blunt head trauma. Ann Emerg 479 Med 2013;62:597-603.

480 19. Babl FE, Borland M, Ngo P, et al. Paediatric Research in Emergency Departments

481 International Collaborative (PREDICT): first steps towards the development of an Australian and 482 New Zealand research network. Emerg Med Australas 2006;18:143-7.

483 20. Babl FE, Lyttle MD, Bressan S, et al. A prospective observational study to assess the 484 diagnostic accuracy of clinical decision rules for children presenting to emergency departments 485 after head injuries (protocol): the Australasian Paediatric Head Injury Rules Study (APHIRST). 486 BMC Pediatr 2014;14:148.

487 21. Hodges JL, Lehmann EL. Estimates of location based on rank tests. The Annals of 488 Mathematical Statistics 1963;34:598-611.

489 22. Badawy MK, Dayan PS, Tunik MG, et al. Prevalence of brain injuries and recurrence of 490 seizures in children with posttraumatic seizures. Acad Emerg Med 2017;24:595-605.

491 23. von Elm E, Altman DG, Egger M, Pocock SJ, Gøtzsche PC, Vandenbroucke JP.

492 Strengthening the reporting of observational studies in epidemiology (STROBE) statement:

493 guidelines for reporting observational studies. Br Med J 2007;335:806-8.

This article is protected by copyright. All rights reserved 
Patient Observation and Cranial Computed Tomography Use

494 24. Babl FE, Oakley E, Dalziel SR, et al. Accuracy of clinician practice compared with three

495 head injury decision rules in children: a prospective cohort study. Ann Emerg Med 2018;71:703-

49610.

497 25. Lockie FD, Dalton S, Oakley E, Babl FE, Paediatric Research in Emergency Departments

498 International C. Triggers for head computed tomography following paediatric head injury:

499 Comparison of physicians' reported practice and clinical decision rules. Emerg Med Australas

$500 \quad 2013 ; 25: 75-82$.

501 26. Stanley RM, Hoyle JD, Jr., Dayan PS, et al. Emergency department practice variation in

502 computed tomography use for children with minor blunt head trauma. J Pediatr 2014;165:1201-6

$503 \mathrm{e} 2$.

504 27. Forero R, Man N, Ngo H, et al. Impact of the four-hour National Emergency Access Target

505 on 30 day mortality, access block and chronic emergency department overcrowding in Australian

506 emergency departments. Emerg Med Australas 2019;31:58-66.

507 28. Jones $\mathrm{P}$, Wells $\mathrm{S}$, Harper A, et al. Impact of a national time target for ED length of stay on

508 patient outcomes. N Z Med J 2017;130:15-34.

509 29. Hess EP, Homme JL, Kharbanda AB, et al. Effect of the head computed tomography choice

510 decision aid in parents of children with minor head trauma: a cluster randomized trial. JAMA

511 Netw Open 2018;1:e182430.

512 30. Nigrovic LE, Stack AM, Mannix RC, et al. Quality improvement effort to reduce cranial

513 CTs for children with minor blunt head trauma. Pediatrics 2015;136:e227-33.

514 31. Coon ER, Newman TB, Hall M, Wilkes J, Bratton SL, Schroeder AR. Trends in imaging

515 findings, interventions, and outcomes among children with isolated head trauma. Pediatr Emerg

516 Care [published online ahead of print April 24, 2018].

517 32. Jennings RM, Burtner JJ, Pellicer JF, et al. Reducing head CT use for children with head

518 injuries in a community emergency department. Pediatrics 2017;139:e20161349.

519 33. Dayan PS, Ballard DW, Tham E, et al. Use of traumatic brain injury prediction rules with

520 clinical decision support. Pediatrics 2017;139:e20162709.

521

This article is protected by copyright. All rights reserved 
Figure. Patient flow diagram

ED, emergency department; GCS, Glasgow Coma Scale; CT, computed tomography; ciTBI, clinically important traumatic brain injury

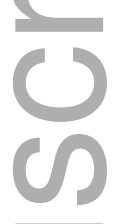

Table 1. Children with minor head trauma (GCS $\geq 14)$ : PECARN TBI risk groups.

\begin{tabular}{|c|c|c|}
\hline PECARN TBI Risk Group & Children Age <2years & Children Age $\geq 2$ years \\
\hline \multirow[t]{2}{*}{ High } & Altered mental status* & Altered mental status* \\
\hline & Palpable skull fracture & Signs of basilar skull fracture ${ }^{\S}$ \\
\hline \multirow[t]{4}{*}{ Intermediate } & Severe injury mechanism ${ }^{\mathbb{I}}$ & Severe injury mechanism ${ }^{\mathbb{I}}$ \\
\hline & History of loss of consciousness $\geq 5$ secs & History of any loss of consciousness \\
\hline & Non-frontal scalp hematoma & History of vomiting \\
\hline & Not acting normally per parents & Severe headache in the ED \\
\hline Very Low & None & None \\
\hline \multicolumn{3}{|c|}{$\begin{array}{l}\text { GCS, Glasgow Coma Scale; PECARN, Pediatric Emergency Care Applied Research Network; TBI, traumatic brain injury; ED, } \\
\text { emergency department }\end{array}$} \\
\hline
\end{tabular}


${ }^{\mathbb{I}}$ Motor vehicle crash with patient ejection, death of another passenger, or rollover; pedestrian or bicyclist without helmet struck by a motorized vehicle; falls of higher than 3 feet for children younger than 2 years of age and higher than 5 feet for children older than 2 years of age; or head struck by a high-impact object.

Table 2. Patient demographics, PECARN TBI risk groups and patient outcomes

\begin{tabular}{|c|c|c|c|c|c|c|}
\hline 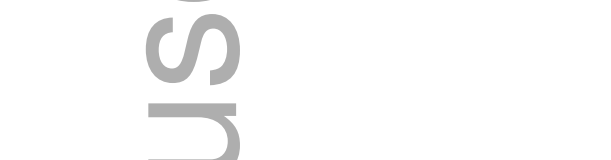 & \multicolumn{2}{|c|}{$\begin{array}{c}\text { Planned observation } \\
\mathrm{n}(\%)\end{array}$} & \multicolumn{2}{|c|}{$\begin{array}{c}\text { No planned observation } \\
\mathrm{n}(\%)\end{array}$} & \multicolumn{2}{|c|}{$\begin{array}{l}\text { Rate Difference } \\
\qquad \%(95 \% \mathrm{CI})\end{array}$} \\
\hline & 4,945 & $(26.8)$ & 13,526 & $(73.2)$ & -46.4 & $(-47.8$ to -44.9$)$ \\
\hline \multicolumn{7}{|l|}{ Demographic characteristics } \\
\hline \multicolumn{7}{|l|}{ Age (years) } \\
\hline Mean (SD) & 6.2 & $(5.1)$ & 5.5 & $(4.4)$ & 0.7 & $(0.6-0.9)^{\mathrm{a}}$ \\
\hline Median (IQR) & 4.5 & $(1.8-10.7)$ & 4.0 & $(1.9-8.1)$ & 0.3 & $(0.2-0.4)^{b}$ \\
\hline Age $<2$ years & 1,393 & $(28.2)$ & 3,566 & $(26.4)$ & 1.8 & $(0.4-3.3)$ \\
\hline Sex [male & 3,102 & $(62.7)$ & 8,671 & $(64.1)$ & -1.4 & $(-3.0$ to 0.2$)$ \\
\hline \multicolumn{7}{|l|}{ PECARN TBI risk group } \\
\hline Very low & 1,630 & $(33.0)$ & 8,201 & $(60.6)$ & -27.7 & $(-29.2$ to -26.1$)$ \\
\hline Intermediate & 2,714 & $(54.9)$ & 4,273 & $(31.6)$ & 23.3 & $(21.7-24.9)$ \\
\hline High & 601 & $(12.2)$ & 1052 & (7.8) & 4.4 & $(3.4-5.4)$ \\
\hline CT performed & 215 & $(4.4)$ & 1,364 & $(10.1)$ & -5.7 & $(-6.5$ to -5.0$)$ \\
\hline \multicolumn{7}{|l|}{ PECARN TBI risk group } \\
\hline Very low: n (within-group \%) & 25 & $(1.5)$ & 138 & $(1.7)$ & -0.2 & $(-0.8$ to 0.5$)$ \\
\hline
\end{tabular}


Patient Observation and Cranial Computed Tomography Use

Intermediate: $\mathrm{n}$ (within-group \%)

High: n (within-group \%)

\section{Patient outcomes}

ciTBI

Neurosurgical intervention

Intubation for $>24$ hours

Hospital admission $\geq 2$ nights with $+\mathrm{CT}$

Death from head injury

\section{PECARN TBI risk group}

Very low: n (within group \%)

Intermediate: n (within group \%)

High: $n$ (within group \%)

\section{TBI on CT}

\section{PECARN TBI risk group}

Very low: $\mathrm{n}$ (within group \%)

Intermediate: $\mathrm{n}$ (within group \%)

High: n (within group \%)

\section{Hospital Admission rate}

Short stay admission

Ward admission

PICU

\section{Return ED visit ${ }^{c}$}

$$
\begin{aligned}
120 & (4.4) \\
70 & (11.7)
\end{aligned}
$$

$20(0.4)$

$2(0.0)$

$\begin{array}{ll}0 & (0.0)\end{array}$

$20 \quad(0.4)$

0

$0 \quad(0.0)$

$13 \quad(0.5)$

$7 \quad(1.2)$

$32(0.7)$

$\begin{array}{ll}0 & (0.0)\end{array}$

$20 \quad(0.7)$

$12(2.0)$

$2,238 \quad(45.3)$

$2,056 \quad(41.6)$

$199 \quad(4.0)$

$3(0.1)$

703 (14.9)
$569 \quad(13.3)$

$657 \quad(62.5)$

$-8.9 \quad(-10.2$ to -7.6$)$

$-50.8 \quad(-54.7$ to -46.9$)$

$26 \quad(0.9)$

$21 \quad(0.2)$

$2(0.0)$

$125 \quad(0.9)$

0

$-0.5 \quad(-0.8$ to -0.3$)$

$-0.1 \quad(-0.2$ to -0.0$)$

$0.0 \quad(-0.0$ to 0.0$)$

$-0.5 \quad(-0.8$ to -0.3$)$

$1 \quad(0.01)$

$-0.01 \quad(-0.04$ to 0.01$)$

$26 \quad(0.6)$

$99 \quad(9.4)$

$-0.1 \quad(-0.5$ to 0.2$)$

$-8.3 \quad(-10.2$ to -6.3$)$

195 (1.4)

$-0.8 \quad(-1.1$ to -0.5$)$

$\begin{aligned} 1 & (0.01) \\ 46 & (1.1) \\ 148 & (14.1) \\ 1,811 & (13.4) \\ 934 & (6.9) \\ 864 & (6.4) \\ 46 & (0.3) \\ 1,457 & (12.0)\end{aligned}$

$-0.01 \quad(-0.04$ to 0.01$)$

$-0.3 \quad(-0.8$ to 0.1$)$

-12.07 (-14.5 to -9.7$)$

$31.9 \quad(30.4-33.4)$

34.7 (33.3 - 36.2)

$-2.4 \quad(-3.1$ to -1.7$)$

$-0.3 \quad(-0.4$ to -0.2$)$

2.8 (1.6-3.9) 


$\begin{array}{lllllll}\text { Missed ciTBI } & 2 & (0.04) & 0 & (0.0) & 0.04 & (-0.02 \text { to } 0.1)\end{array}$

SD, standard deviation; IQR, interquartile range; CI, confidence interval; GCS, Glasgow Coma Scale; PECARN, Pediatric Emergency Care Applied Research Network; TBI, traumatic brain injury; CT, computed tomography; ciTBI, clinically important traumatic brain injury; CI, confidence interval; PICU, pediatric intensive care unit

${ }^{\mathrm{a}}$ Difference between means $(95 \% \mathrm{CI})$

${ }^{\mathrm{b}}$ Difference between medians (95\% CI)

${ }^{\mathrm{c}}$ If had follow-up interview: Planned observation $n=4,773$; No planned observation $n=12,208$

Table 3. Median intervals: planned observation vs. no planned observation

\begin{tabular}{|c|c|c|c|c|c|c|c|c|}
\hline \multirow[b]{2}{*}{ Interval [hours] } & \multicolumn{3}{|c|}{ Planned observation } & \multicolumn{3}{|c|}{ No planned observation } & \multicolumn{2}{|c|}{ Time Difference } \\
\hline & $\mathrm{n}$ & Median & (IQR) & $\mathrm{n}$ & Median & (IQR) & Median & $95 \% \mathrm{CI}$ \\
\hline Injury to ED arrival & 4,892 & 1.3 & $(0.8-2.2)$ & 13,293 & 1.3 & $(0.8-2.9)$ & -0.1 & $(-0.1$ to -0.1$)$ \\
\hline ED arrival to physician evaluation & 4,936 & 0.4 & $(0.2-0.7)$ & 13,502 & 0.5 & $(0.2-1.0)$ & -0.1 & $(-0.2$ to -0.1$)$ \\
\hline \multicolumn{9}{|l|}{ ED LOS [hours] } \\
\hline Overall & 4,935 & 3.7 & $(2.7-5.2)$ & 13,495 & 1.9 & $(1.2-3.1)$ & 1.7 & $(1.6-1.7)$ \\
\hline \multicolumn{9}{|l|}{ PECARNTBI Risk Group } \\
\hline Very low risk & 1,628 & 3.4 & $(2.4-4.5)$ & 8,186 & 1.6 & $(1.1-2.5)$ & 1.6 & $(1.5-1.7)$ \\
\hline Intermediate risk & 2,707 & 3.7 & $(2.8-5.3)$ & 4,261 & 2.2 & $(1.3-3.5)$ & 1.5 & $(1.4-1.6)$ \\
\hline High risk & 600 & 4.4 & $(3.3-7.3)$ & 1,048 & 3.9 & $(2.4-6.4)$ & 0.7 & $(0.4-0.9)$ \\
\hline
\end{tabular}


Patient Observation and Cranial Computed Tomography Use

\begin{tabular}{|c|c|c|c|c|c|c|c|c|}
\hline Obtained & 213 & 6.0 & $(4.0-13.0)$ & 1,360 & 4.9 & $(3.5-7.5)$ & 1.2 & $(0.6-1.9)$ \\
\hline Not obtained & 4,722 & 3.6 & $(2.7-5.1)$ & 12,135 & 1.7 & $(1.1-2.7)$ & 1.8 & $(1.8-1.9)$ \\
\hline \multicolumn{9}{|l|}{ Total LOS [hours] } \\
\hline Overall & 4,934 & 3.7 & $(2.7-5.5)$ & 13,484 & 1.9 & $(1.2-3.3)$ & 1.7 & $(1.6-1.8)$ \\
\hline \multicolumn{9}{|c|}{ PECARN TBI Risk Group } \\
\hline Very low risk & 1,628 & 3.4 & $(2.5-4.7)$ & 8,184 & 1.7 & $(1.1-2.6)$ & 1.6 & $(1.6-1.7)$ \\
\hline Intermediate risk & 2,706 & 3.8 & $(2.8-5.6)$ & 4,259 & 2.2 & $(1.3-3.8)$ & 1.5 & $(1.4-1.6)$ \\
\hline High risk & 600 & 4.6 & $(3.4-10.5)$ & 1,041 & 6.9 & $(2.9-24.9)$ & -1.5 & $(-2.3$ to -0.9$)$ \\
\hline \multicolumn{9}{|l|}{ Cranial CT } \\
\hline Obtained & 213 & 11.8 & $(4.6-22.0)$ & 1,351 & 14.4 & $(4.5-36.6)$ & -0.7 & $(-1.9$ to 0.3$)$ \\
\hline Not obtained & 4,721 & 3.7 & $(2.7-5.2)$ & 12,133 & 1.7 & $(1.1-2.8)$ & 1.8 & $(1.8-1.9)$ \\
\hline
\end{tabular}

ED, emergency department; LOS, length of stay; TBI, Traumatic brain injury; PECARN, Pediatric Emergency Care Applied Research Network; CT, computed tomography; IQR, interquartile range; CI, confidence interval

Table 4. Adjusted likelihood of cranial CT use

\begin{tabular}{|c|c|}
\hline 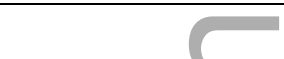 & Total Cohort \\
\hline & $\mathrm{N}=17,969$ \\
\hline & AOR $\quad(95 \% \mathrm{CI})$ \\
\hline Age $\geq 2$ years & Reference \\
\hline$<2$ years & $0.4 \quad(0.4-0.5)^{*}$ \\
\hline Sex (male) & $1.11(0.97-1.3)$ \\
\hline
\end{tabular}

This article is protected by copyright. All rights reserved 
Patient Observation and Cranial Computed Tomography Use

PECARN TBI risk

Very low

Intermediate

High

History of seizure

Time from injury (hours)

\section{Observation status}

Not observed

Planned observation

Year

2011

2012

2013

2014
Reference

$$
\begin{array}{cl}
8.1 & (6.7-9.7)^{*} \\
77.0 & (62.8-94.5)^{*} \\
4.6 & (3.2-6.5)^{*} \\
1.03 & (1.02-1.04)^{*}
\end{array}
$$

\section{Reference}

$$
0.2(0.1-0.2)^{*}
$$

\section{Reference}

$0.9 \quad(0.6-1.2)$

$0.8 \quad(0.6-1.0)$

$0.8 \quad(0.6-1.0)$

Clustered by hospital site; ${ }^{*} \mathrm{p} \leq 0.001$

CT, computed tomography; AOR, adjusted odds ratio; CI, confidence interval; PECARN, Pediatric Emergency Care Applied Research Network; TBI, traumatic brain injury

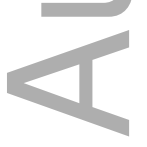

This article is protected by copyright. All rights reserved 

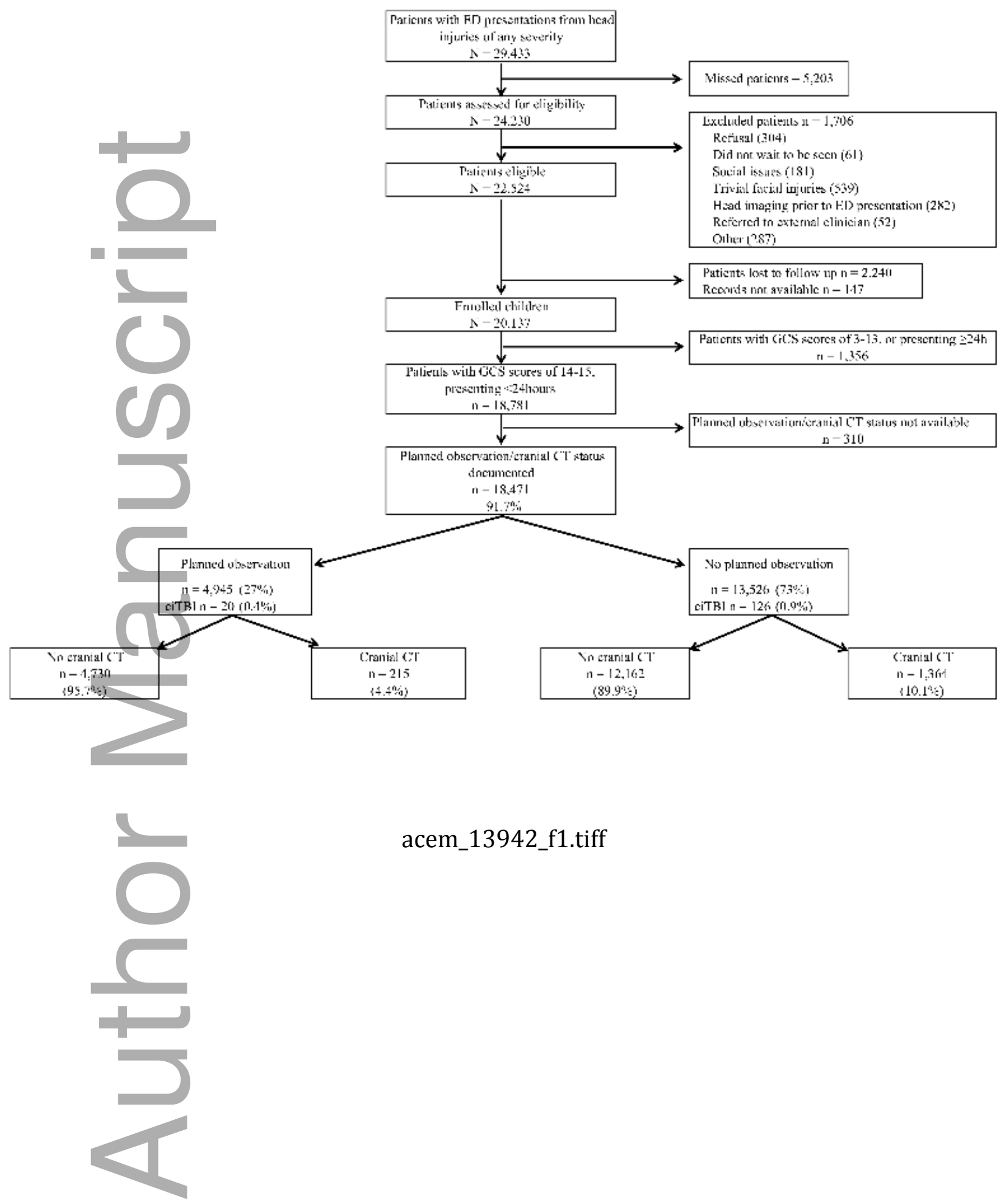

acem_13942_f1.tiff 


\section{University Library}

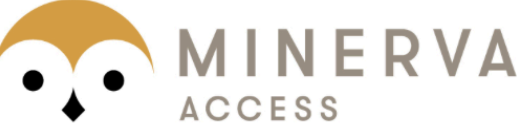

A gateway to Melbourne's research publications

Minerva Access is the Institutional Repository of The University of Melbourne

\section{Author/s:}

Singh, S;Hearps, SJC;Borland, ML;Dalziel, SR;Neutze, J;Donath, S;Cheek, JA;Kochar, A;Gilhotra, Y;Phillips, N;Williams, A;Lyttle, MD;Bressan, S;Hoch, JS;Oakley, E;Holmes, JF;Kuppermann, N;Babl, FE

Title:

The Effect of Patient Observation on Cranial Computed Tomography Rates in Children With Minor Head Trauma

Date:

2020-03-26

Citation:

Singh, S., Hearps, S. J. C., Borland, M. L., Dalziel, S. R., Neutze, J., Donath, S., Cheek, J. A., Kochar, A., Gilhotra, Y., Phillips, N., Williams, A., Lyttle, M. D., Bressan, S., Hoch, J. S., Oakley, E., Holmes, J. F., Kuppermann, N. \& Babl, F. E. (2020). The Effect of Patient Observation on Cranial Computed Tomography Rates in Children With Minor Head Trauma. ACADEMIC EMERGENCY MEDICINE, 27 (9), pp.832-843. https://doi.org/10.1111/ acem. 13942.

Persistent Link:

http://hdl.handle.net/11343/275567 